\title{
An Experiential Learning in Web Technology Course
}

\author{
Vijayakumar Bhajantri ${ }^{1}$,Sujatha $\mathrm{C}^{2}$, Shilpa Yaligar ${ }^{3}$, Manjula K.Pawar ${ }^{4}$ \\ ${ }_{1,2,3,4}$ Dept. of Computer Science and Engineering, \\ B.V. Bhoomaraddi College of Engineering and Technology, \\ Hubballi, Karnataka,India \\ vijaybhajantri@bvb.edu',sujata_c@bvb.edu², shilpa_yaligar@bvb.edu ${ }^{3}$, manjulap@bvb.edu ${ }^{4}$
}

\begin{abstract}
Web Technology is the development of the mechanism that allows two of more computer devices to communicate over a network. To understand such a complex system there is a need of experiential learning. Authors adopted an "Experiential learning" for teaching the web technology course. Teaching with a hands-on session where the teacher explains the concepts with demonstration and students simultaneously practice\& also modify the fields to observe the change. After the end of chapter the students learning is verified by giving the structured enquiry category assignments and are evaluated. At the end of delivery of course i,e one month before the term end the collective learning is verified through the course project. Two course projects are carried out by the students as a team of two. One is the intranet project, where student understands how to connect and work in a local area network and the other is internet project, where student learns how to host a website globally. In both the projects students worked on LINUX platform with LAMP as a framework and developed web apps using HTML5, CSS 3,JQuery,PHP and SQL.
\end{abstract}

\section{Vijayakumar Bhajantri}

Dept. of Computer Science and Engineering,

B.V. Bhoomaraddi College of Engineering and Technology,

Hubballi, Karnataka,India

vijaybhajantri@bvb.edu
The experiential learning enhanced the student's domain knowledge of Linux architecture, computer networks, and web and database servers applying in real time scenarios. Finally we conclude such practical/programming oriented courses should be designed as hands-on lab courses

Keywords : Experiential learning, intranet, internet, three tier architecture, projects

\section{Introduction}

In the previous curriculum the credits of Web Technology(WT) course was distributed as three credits for theory and 1.5 credits for laboratory. The course instructors and students felt that the lecture session and the practical session did not synchronize the content and felt that demonstration with simultaneous practice would make the students learn and understand the concepts better. As per the feedback from both, the course is redesigned as a hands-on lab course of 2.5 credits. So we propose to adopt an experiential learning forteaching this course.

Authors propose and adopt an "Experiential Learning" for teaching the web technology course. A hands-on session is followed where the teacher explains the concepts with demonstration and students simultaneously practice \& also modify the fields to observe the change. Student's concept learning is verified by evaluating them on exercises and structured enquiry type of assignments and the collective learning is verified through course project. 
Two course projects are carried out by students one is internet project developed individually and another intranet with three tier architecture as a team of two.

The paper is further organized as follows: Section II describes the literature survey. Section III narrates the proposed teaching methodology. The assessment and result analysis is discussed in Section IV along with evaluation parameters and rubrics and finally conclude in section $\mathrm{V}$.

\section{Literature Survey}

This section discusses about the existing practices in teaching and conducting programming courses for undergraduates.

In literature hardly we find contributions in teaching this course. Only Yu Lijie et al proposed a new teaching method named as Learning-ByDoing[1]. It adopts the interactive lecture style, while simultaneously using a Training Kit Program integrated the theoretical knowledge with practical examples named example-based paradigms. Students developed web driven database application to address the information needs of a business enterprise. The following arethe contributions of traditional education models.

The traditional education model is based on verbal lecture, drill and practice sessions, printed handouts, structured classroom activities, and office hours [2]. In its pure form this model is grounded in thebelief that knowledge is objective and the purpose of the teaching process is to transfer this static body of knowledgefrom its source (teacher, textbook, etc.) to the student [3]. The student, using this point of view, is seen as a passivelearner "waiting to be filled" with knowledge, but theknowledge is not static. A fundamental shift is occurring in education as aresult of the increasing use of computers [4]. Lee and Lehman [5] have emphasized the active role learner's mustplay in order to learn in hypertext-based learning environments.

\section{Implementation}

We propose a methodology that helps students understand engineering concepts and go beyond the knowledge level to higher levels of thinking. It also helps them to apply, analyze, and synthesize, to create new knowledge, and solve new problems. So we teachers need to recognize our challenge to go beyond knowledge about effective teaching. We need to apply these strategies, analyze what works, and take action to modify or synthesize our learning's to help our students learn in a way that works for us as individuals and teams of teachers.

We proposed an approach called as "Experiential Learning" as shown in Fig.3.1. It adopts the interactive lecture style, compiled effective material for all batches, regular effective assessment method (exercise and structured enquiry) and course project with added weightage given to course project. Course project is major change in the process of Experiential Learning i.e. students are exposed to real-time web services by hosting their own websites on commercial web servers by coding and understanding.

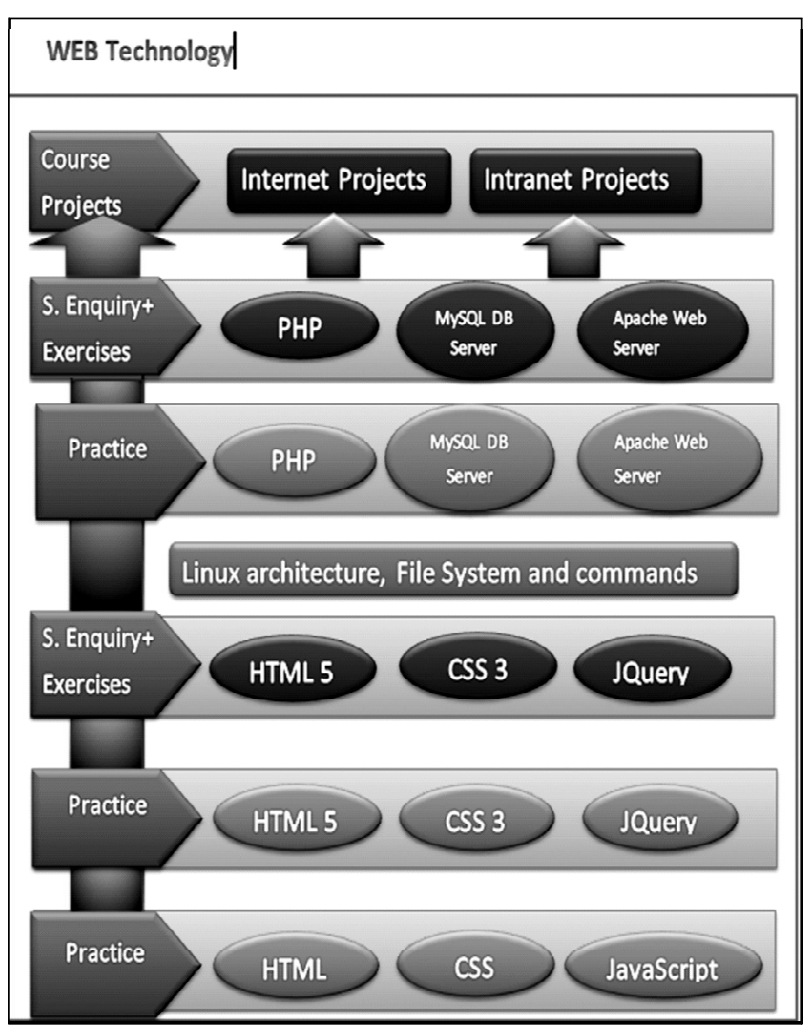

A. COURSEPROJECT

Course projects help to reinforce the students' theoretical knowledge and develop their ability to apply this knowledge to the solution of practical problems. It prepares them for their diploma project and for independent work in their chosen field and promotes the development of their creative abilities.

The web-technology course is a blend of HTML5, CSS3, JQuery, PHP and XML.We used LAMP framework for developing and deploying course 
projects. Rigorous practice, exercise and structured enquiry are carried out. We have allotted course project into two themes: i) Intranet ii) Internet.

Intranet is a local or restricted communications network, especially a private network created using World Wide Web software. An intranet uses TCP/IP, HTTP, and other Internet protocols and in general looks as a private version of the Internet.

The idea behind this category of project is to make most of the department works digitalized. The brainwave for this thought is from Honorable Prime MinisterN.D. MODI's "DIGITAL INDIA" concept. Project topics are chosen in concern to help department in maintaining the documents and access them online. To implement this category of projects students are exposed to three-tiered-architecture system which is shown in Fig. 3.2.

The three tiers in three-tier architecture are:

Presentation Tier: Occupies the top level and displays information related to services available on a website. This tier communicates with other tiers by sending results to the browser and other tiers in the network.

Application Tier: Also called the middle tier, logic tier, business logic tier, this tier is pulled from the presentation tier. It controls application functionality by performing detailed processing.

Data Tier: Houses database servers where information is stored and retrieved. Data in this tier is kept independent of application servers or business logic.

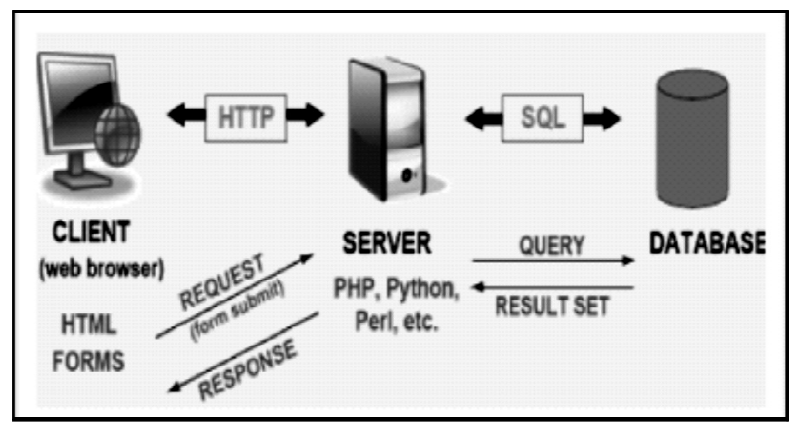

Fig. 3.2Three-Tier Web Architecture

IP addresses of the machines are used to make intranet connection, which are identified by the command "ifconfig".Two or more software's or running processes communicate through their software ports.

DHCP protocol: Dynamic Host Configuration Protocol, a protocol for assigning dynamic IP addresses to devices on a network. With dynamic addressing, a device can have a different IP address every time it connects to the network. Dynamic addressing simplifies network administration as the software keeps track of IP addresses rather than requiring an administrator to manage the task. Since BVBCET cyber roam server has been set to DHCP method for acquiring IP address i.e. every 24 hours, IP address of each machine will change automatically. Student has to use appropriate method while developing software to get IP address. This will expose students to real time network protocols.

The Internet is a global system of interconnected computer networks that use the standard Internet protocol suite(TCP/IP) to link several billion devices worldwide. It is a network of networks that consists of millions of private, public, academic, business, and government networks of local to global scope, linked by a broad array of electronic, wireless, and optical networking technologies. The Internet carries an extensive range of information resources and services, such as the inter-linked hypertext documents and applications of the World Wide Web (WWW), the infrastructure to support email, and peer-to-peer networks for file sharing and telephony.

The World Wide Web is an information system of interlinked hypertext documents and other digital resources that are accessed via the Internet. It has become known simply as the Web. Hypertext documents are commonly called web pages, which are primarily text documents formatted and annotated with theHypertext Markup Language (HTML). Webpages may contain links to images, video, and software components that are rendered to users of a web browserapplication, running on the user's computer, as coherent pages of multimedia content. Embedded hyperlinks permit users to navigate between web pages. When multiple web pages are published with a common theme or within a common domain name, the collection is usually called a web site.

It category includes design and hosting of personal website by each student individually. Students can either adopt the method of hard coding or can make use of HTML5 templates. The idea behind adopting this theme is to showcase the skills acquired by

\section{JEE E्:}


students in a squashed way. Making them design and host their own website is the best way to expose them to the digital world.

Benefits of having one's personal website:

- Nowadays, prospective employer and recruiters take advantages of the internet to do the initial search for job applicant's information. They want to know more about the applicants their skills, passion and life. Having a great website will greatly increase the chance of being selected for an in-depth interview.

When building their own website they need to understand every aspect involved, from domain registration, hosting, web design, front-end coding, back-end coding, usability, search engine optimization, conversion optimization.

\section{Steps to Host A Website}

\section{i. SETUP}

a. Register domain names in the infocell website (http://infocell.bvb.edu) with ".in" as domain.

b. Domains are registered under Godaddy website (http://www.godaddy.in) hosting.

c. Create account in hosting server.

d. Map IP address/domain names to hosting server IP address.

e. Create FTP account in hosting server.

f. Create DB account to access MySQL DB remotely.

ii. Design and Implement website

iii. Dump files into server and Host website

a. Individual website files are stored in GoDaddy commercial shared web server.

b. Accesswebsites through domain name by any browser.

c. Access shared web server remotely by using Filezilla software (Edit, update, delete).

d. Access MySQL DB server and to execute SQL Workbench software is used.

e. Access MySQL DB server remotely assign the grants by executing the following "GRANT ALL PRIVILEGESON **TO 'root`@ \%”;“”

Filezilla: FileZilla is free software, cross-platform FTP application, consisting of FileZilla Client and FileZilla Server. Client binaries are available for Windows, Linux, and Mac OS X, server binaries are available for Windows only. The client supports FTP, SFTP and FTPS (FTP over SSL/TLS). Support for SFTP (SSH File Transfer Protocol) is not implemented in FileZilla Server.

\section{Observations}

During the development of course project, they have to write their own logic for real time problem, the students are challenged to develop a web driven database application to address the information needs of a business enterprise. Students are responsible for designing a solution to that e-business problem, and implementing and documenting their solution, those get a marked evaluation

\section{A. Students Response}

Our experience with this course is, on balance, highly constructive. Almost, with some exceptions, every student expressed gratitude for having been able to take an entire system through design, implementation, operation, and documentation, and have often told the instructor that this course is exactly the kind of training they should have, and most have rated the class among the most valuable course they have completed. The program provides undergraduate students with valuable opportunities to acquire not only knowledge but also experience and skills in the web technology areas, motivating them for careers and graduate studies in these areas.

Students have well appreciated our contribution to the project. We think no, because inside this approach:

(1) The educational process is different (there is the presence of a good interaction student -teacher);

(2) The role of the students is active (they build their knowledge working in a real context);

(3) Balance between knowledge and skills/competency development and integrates both student performance and outcomes assessment. 
The feedback students provide about your teaching in their semester course evaluations can be valuable in helping you improve and refine your teaching. Eliciting mid-semester student feedback has the additional benefit of allowing you to hear your students' concerns while there is still time in the semester to make appropriate changes

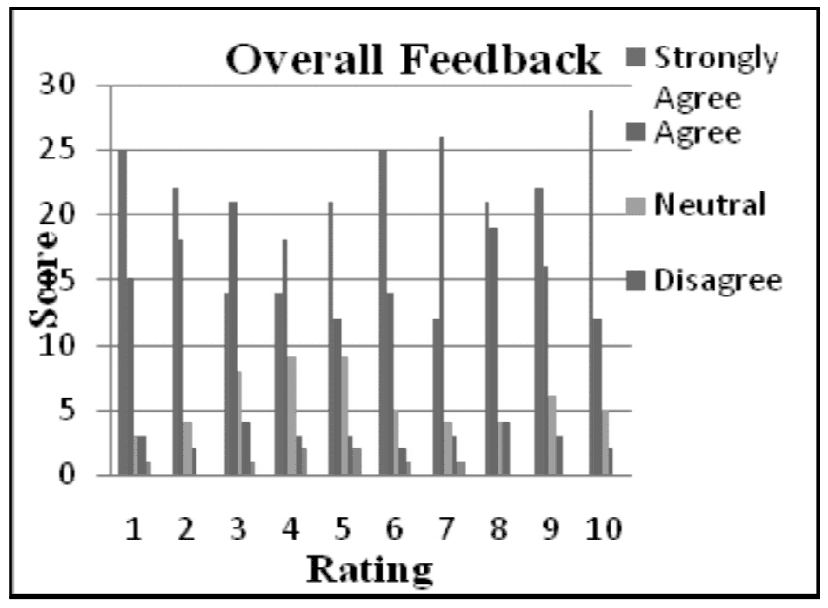

Fig. 4.1: Feedback Analysis

The Effectiveness of the course by experiential learning approach is measured with students' feedback. We have considered 10 questions for feedback that focus on all learning aspects of the course. We analyzed the effectiveness with the bar graph as shown in the Fig.4.1 above which is a plot of score against the ranks(ratings). From the graph we can infer that we have got strong response for option 'Strongly Agree' of all the questions from thestudents, followed by options Agree, Neutral, Disagree, and Strongly Disagree.

We consider the response of the students for questions 1, 2 and 5 individually which mainly focus on the technical knowledge aspect of the course. Students have given most positive feedback for these questions. On an average more than $80 \%$ students have given positive feedback for all ten questions.

\section{Outcomes And Assessment Methods}

The program outcomes attained in Web Technology course is listed in the Table 4.1. Still we need to enhance knowledge in using tools for design and testing modules for different browsers and platforms as few features of html5 are not supported by some browsers.
Table 4.1 PO mapped

\begin{tabular}{|c|c|c|}
\hline Category & PO & $\begin{array}{l}\text { Outcomes } \\
\text { (students were able to) }\end{array}$ \\
\hline $\begin{array}{l}\text { Intranet } \\
\text { and } \\
\text { Internet } \\
\text { course } \\
\text { projects }\end{array}$ & $\begin{array}{l}\mathrm{a} \\
\mathrm{b} \\
\mathrm{c} \\
\mathrm{k}\end{array}$ & $\begin{array}{l}\text { Identify Inputs, outputs, } \\
\text { constraints and } \\
\text { assumptions. } \\
\text { Test the system/ process/ } \\
\text { component / program for } \\
\text { its functionality and } \\
\text { performance } \\
\text { Apply the knowledge of } \\
\text { chosen tool / technique to } \\
\text { perform the chosen } \\
\text { engineering activity. }\end{array}$ \\
\hline
\end{tabular}

\section{A. RESULTS AND ANALYSIS}

The Fig: 4.2 show the comparison of results and analysis of this year and previous two batches. Thisshows that conduction of experimental web technologies has better results compared to previous two batches. There is better improvement in $\mathrm{S}$ grade. This shows that experimental learning made students to improve their web development skills.

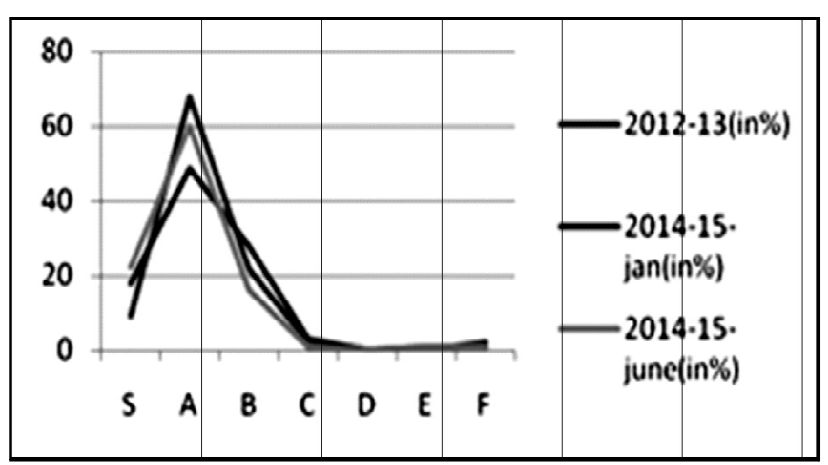

Fig. 4.2 Results and Analysis

\section{Conclusion}

In this paper we have proposed the new methodologyExperiential Learning in Web technology course. The real time course project-based methods are effective techniques for web program learning. The main theme of the methodology behind was, a series of program examples had been developed to enhance teaching and learning of web application development, it was easy to use and the step-by-step feature helped students gain 
understanding of web application developing process. The project centric experiential learning of this course is Internet and Intranet course projects which were carried out as individual and group of students respectively. This approach enhanced student knowledge in developing real time web application and hosting on commercial server. The delivery of this teaching approach has been a real learning experience even for the authors and suggests that such programming oriented courses should be designed and delivered as hands-on lab courses.

\section{References}

[1] Yu Lijie, Xia liwen, Guo Wenrong, "Learning By Doing", Second International Conference on Education Technology and Training, 2009.

[2] R.J. Spiro, P.J. Feltovich, M.J. Jacobson, R.L.Coulson, "Cognitive Flexibility, constructivism and hypertext: Random access instruction for advanced knowledge acquisition in ill-structured domains", Duffy T., D. and D. Jonassen (Eds.), Constructivism and the technology of instruction, Hillsdale, NJ: Erlbaum, 1992
[3] J.M. Lightfoot, "Designing and Implementing a 'Full-Service' ClassPage on the Internet", Journal of Educational Multimedia and Hypermedia, vol. 9,2000.1

[4] A. Rath, W.A. Rieck, and D. Wadsworth, "Educator's Approaches to Multimedia CDROM Development: Programming Processes and Curricular Concepts", Journal of Technology and Teacher Education, 1998.6, pp. 205 -220.

[5] J.H. Strange, "A cultural revolution: From books to silver disks", Metropolitan Universities, 1995.6, pp.39-51

[6] Y.B. Lee, J.D. Lehman, "Instructional cuing in hypermedia: A study with active and passive learners", Journal of Educational Multimedia and Hypermedia, 1993.2, pp.25-37. 